\title{
Nomadic Relay-directed Joint Power and Subchannel Allocation in OFDMA-based Cellular Fixed Relay Networks
}

\author{
Mohamed Salem ${ }^{\natural}$, Abdulkareem Adinoyi ${ }^{\natural}$, Halim Yanikomeroglu ${ }^{\natural}$, and Young-Doo Kim ${ }^{b}$ \\ ${ }^{\natural}$ Department of Systems and Computer Engineering, \\ Carleton University, Ottawa, Canada \\ ${ }^{b}$ Samsung Electronics, SAIT, Korea
}

\begin{abstract}
Various standardization activities leading to $4 \mathrm{G}$ and beyond networks have considered the synergy of OFDMA and multihop relaying thus giving way for the fixed relay station (FRS)-based radio access network. As such, the next-generation networks will comprise a plethora of performance enhancing devices among which is the plug-and-play nomadic relay station (NRS). It is essential for such dense and inevitably frequency reuse-aggressive networks to employ efficient mechanisms to mitigate the co-channel interference and to provide prudent energy utilization; this brings about the timely environmental concerns and the so-called green wireless initiative in designing future wireless networks. We present a novel joint power and subchannel allocation algorithm for the emerging OFDMA-based nomadic-augmented fixed relay networks. This NRS-directed algorithm performs adaptive power control (APC) within the autonomous opportunistic NRS medium access and channel reuse, using two different approaches. The APC mechanism is realized in an open-loop manner requiring no feedback from the WT. We demonstrate the power savings and user throughput improvement obtained through the joint scheme. We also identify a throughput-power saving trade-off in terms of the number of deployed FRSs. Through this work, the authors further establish their pioneering techniques for realizing the concept of NRSaugmented networks.
\end{abstract}

Index Terms- OFDMA, RRM, multihop, relaying, nomadic, power control, intra-cell reuse, dynamic routing, scheduling, cooperation.

\section{INTRODUCTION}

Power control (PC) is an important interference combatting mechanism thereby constituting a means for improving the network performance through enabling efficient utilization of system resources. Traditionally, PC has been employed for combatting co-channel and adjacent-channel interferences in multi-cellular networks. The co-channel interference (CCI) due to frequency reuse is one of the most limiting factors on wireless system capacity. In fact, the radio resource management (RRM) schemes earmarked for the future wireless networks are designed to be aggressive in frequency reuse. Mainly due to the fact that licensed radio spectrum is scarce whereas a plethora of wireless devices will form an integral part of the future network architecture. Devices ranging from nomadic relays [1] to femtocell access points will be utilizing the orthogonal frequency division multiple access (OFDMA) technique and contending for radio resources.

The nomadic relay station (NRS) is a plug-and-play wireless device; an idea that has been entrenched in the IEEE 802.16

Patent filings from this work have been made by Samsung Electronics Co. Ltd., SAIT, Korea. Patent application no: P2009-0084026. E-mail:\{mrashad, adinoyi, halim\}@sce.carleton.ca,yd76.kim@samsung.com. standards [2], and gaining widespread acceptance. NRSs are technically stationary devices but portable and thus batterypowered. In the survey paper [1], motivational scenarios for using mobile multihop relaying are provided. Therein, NRSs can be deployed to provide a temporary coverage and capacity in an area where FRSs may not provide the required QoS such as in trade fairs and sporting events or in disaster recovery situations. In addition, NRSs can be used to spread the capacity in a large building. In such cases, NRSs will coexist with FRSs yet with potentially much better communication links to the wireless terminals (WTs). To the extent of our knowledge, no work so far has provided mechanisms for integrating the autonomous NRSs into the cellular network or suggested the underlying RRM schemes and protocols to facilitate their coexistence with FRSs. Since the current literature does not cover the systems and architecture considered in this paper, in the following we briefly review some relevant works in the literature of decentralized and iterative PC.

Centralized PC schemes require reliable measurements of the gains in all radio links in the system. For practical implementation, PC schemes have to rely on far less accurate measurements and limited information to enable distributed operation. A common experience is that, a simple proportional control algorithm, which increases the transmitter power in a link if the received signal-to-interference-plus-noise ratio (SINR) is too low and decreases it when the SINR is high, will normally work well [3]. Thus, such an approach can be viewed as a gateway to distributed PC, where a transmitter utilizes limited knowledge for power adaptation. Towards that end, [4] develops distributed iterative PC algorithms that utilize available measurements and converge in stochastic sense, in contrast to deterministic PC schemes that rely on exact or perfect control quantities (SINR or interference). In [5], the authors consider iterative PC as part of the downlink (DL) schemes employing a non-orthogonal amplify-and-forward cooperative protocol. Each BS independently re-computes user received inter-cell interference as a function of the transmit power values in the previous iteration.

It is known that PC can provide: 1- Means to prevent receiver saturation when the transmitter is too close. 2- Energy savings. Here, the green radio initiatives may readily come to mind where, for instance, low-consumption terminals that depend on solar energy have been developed and showcased in the Mobile World Congress 2009 [6]. 3- Throughput improvement under frequency reuse through reducing the 


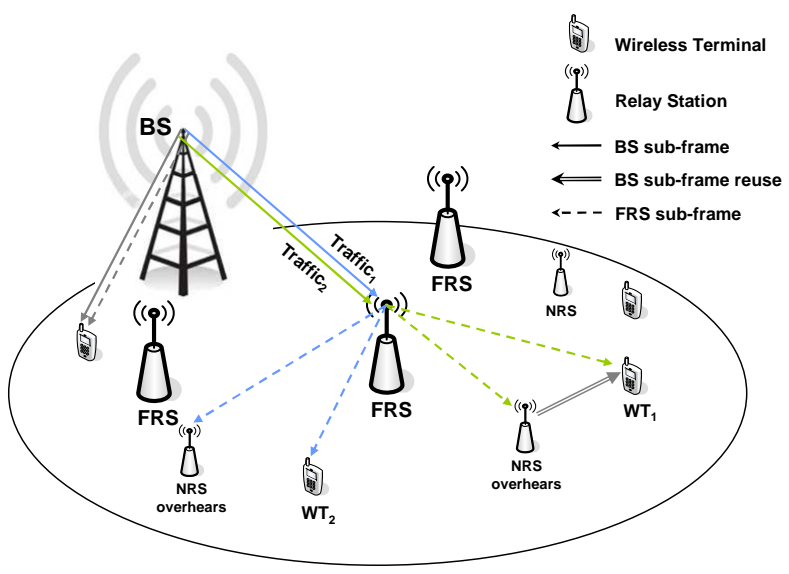

Fig. 1. The region of interest may represent a cell of the hexagonal grid, a sector of a cell, or a 'cell' of the LTE-A eNB in the multicellular network. All resources are available in this region.

associated CCI. It will be desirable therefore for a transmitting node, especially if battery-powered, to utilize the minimum energy possible to achieve the desired QoS. In this work, we investigate the DL operation of a visionary wireless network model, where FRSs are augmented by NRSs with the aim of providing a more reliable cost-effective service. These NRSs will be often acting as intermediate nodes between a serving FRS and a WT forming a three-hop communication. To alleviate the burden on system resources due to multihop relaying, intelligent RRM schemes are needed to facilitate aggressive resource reuse and meanwhile combat the potential CCI through opportunistic reuse and interference avoidance mechanisms. Device power utilization could be particulary critical, in nomadic relay-augmented networks, where the NRS acts as an extension of the WT. The authors devised a smart technique to enable autonomous medium access and aggressive reuse through NRSs for such networks in [7]. The contributions in this paper can be summarized as follows:

- We present a novel NRS-directed joint power and subchannel allocation algorithm for the emerging OFDMAbased nomadic-augmented fixed relay networks. The algorithm performs adaptive power control (APC) within the autonomous opportunistic NRS medium access and channel reuse, using two different approaches, to assist a troubled WT through cooperation with the serving FRS.

- The APC mechanism is realized in an open-loop manner requiring no feedback from the assisted WT; significant gains have been achieved through NRS assistance at no additional cost.

- The performance returns in terms of power savings and user throughput are evaluated in a realistic environment considering non-ideal NRS-WT links.

- We identify a throughput-power saving trade-off in terms of the number of deployed FRSs.

- While no other work in the literature has addressed such systems and architecture, we further sustain the concept of nomadic relay-augmented fixed relay networks we previously established in [7].

\section{The NeTwORK OF FIXED AND NOMADIC RELAYS}

Since the radio resource allocation (RRA) schemes for the BS and the FRSs are not the focus of this paper, in this section we follow the style in [7] to describe the essential details of the underlying system. A representative partial network of the proposed system is shown in Fig. 1. The figure demonstrates the DL transmissions over two consecutive equal-length sub-frames and the half-duplex operation of all relay stations. The system operates in time-division duplexing (TDD) mode. An FRS receives only during the first subframe (BS sub-frame) and transmits during the second (FRS sub-frame). There are $M$ FRSs deployed in the considered region at strategic locations. Through a dynamic WT-based routing (or link selection) strategy, the WT selects one access link out of $M+1$. The basic allocation unit is the OFDM subchannel comprising a number of adjacent subcarriers; there are $N=|\mathcal{N}|$ data subchannels for each the channel fading is flat. All resources are available in each region similar to that shown in Fig. 1. Adaptive modulation (AM) is employed only at BSs and FRSs so that the achievable rate of the link from source $m$ to destination $k$ at a particular target bit error rate $P_{e}$ is a function of the OFDM subchannel bandwidth $W$ and the received SINR $\gamma_{m, k, n}$, considering the CCI observed in the previous DL frame as in (1) [8]. In our results, the achievable spectral efficiency is upper bounded by $10 \mathrm{bps} / \mathrm{Hz}$.

$$
R_{m, k, n}=W \log _{2}\left(1+\frac{-1.5 \gamma_{m, k, n}}{\ln \left(5 P_{e}\right)}\right) .
$$

There are $K$ active WTs in the network and $K_{\text {nom }}$ NRSs. We have considered the case in which $K_{n o m}=K$ to reflect on a scenario where each WT has placed, or selected out of many, an NRS that is dedicated to assist it on demand. The NRS is thus stationary, in a close vicinity of the WT, and often has a very good connection to the WT; likely a line-of-sight. The NRS could be larger than mobile-phone kind of terminals and thus can employ more antennas to strengthen its link to either the serving FRS or the WT through beam steering. Note that the potential serving FRSs are stationary, while the NRS can be equipped with the computational power for its beam to follow a mobile WT. That is because the NRS does not handle upper layer issues such as applications and human interfaces. Fixed power allocation for only BSs and FRSs is assumed. Continuous backlog is assumed only at the BS buffers. The basic operation of the system can be described as follows. In the BS sub-frame, the BS transmits to the direct WTs (dWTs) and the FRSs with connected WTs while any NRS can access one or more subchannels at a random instant, immediately after listening to the activity on all subchannels, and transmit to a particular WT during the remaining time of the same subframe. Intra-cell (or intra-region) reuse occurs if the acquired subchannel is allocated to a BS-FRS feeder link or a BSdWT access link. In the FRS sub-frame, the BS continues to communicate with the dWTs on the same subchannels; all channels are invariant over the DL time frame. FRSs transmit to their connected WTs while the NRSs overhear and always decode the transmissions to their respective terminals. An NRS may not overhear and transmit concurrently. For the sake of illustration, we consider a protocol where NRS cooperates with only FRSs. Therefore, the NRS transmits during BS sub-frame $i+1$, after receiving from the FRS during FRS sub-frame $i$. The advantages of such protocol over ARQ through the serving FRS are discussed in [7] in terms of latency, resource efficiency, and spatial diversity. 


\section{NRS OPERATIONS AND PC MECHANISM}

\section{A. NRS Subchannel Acquisition and Selective Transmission}

The NRS chooses a random instant to listen to the whole bandwidth and estimates, without detection, the total received power on each subchannel. Based on the proximity of the NRS to the WT, such received power on a subchannel at this instant can approximate the interference level that could be observed by the WT if the NRS uses that subchannel for the remaining sub-frame duration. Due to the asynchronous access, the probability of more than one NRS listening at the same instant, and furthermore, interfering significantly with each other, is negligible. The asynchronous access allows the NRS to sense the activity of other nearby NRSs, or more precisely, with high interfering-link gains; thus minimizing the likelihood of choosing the same subchannels immediately acquired by such NRSs. A similar idea has been employed for ad-hoc networks in [9] where a cluster head estimates the interference it will cause to others when it uses a particular band relying mainly on channel reciprocity. Such condition is unnecessary in our schemes since the NRS only listens to estimate the interference that its WT will experience on a subchannel if the NRS uses it. Figures 2 and 3 differentiate between the two proposed approaches through which the number of acquired subchannels, $N_{r}$, can be determined given that the NRS attempts to minimize latency by forwarding all the requested and reliably detected data segments during the remaining BS sub-frame duration while maintaining their AM modes. While the 'data segment' on which the NRS selective transmission operates may generally refer to a packet or a subchannel, we here consider a subcarrier as the data segment as shown in Fig. 2 and Fig. 3. By selective transmission, the NRS potentially requires a fraction of the resource used by the FRS; this is particularly useful since NRSs only access the channel over a fraction of the BS sub-frame.

\section{B. NRS Joint Power-Subchannel Allocation Algorithm}

While it is known that only marginal gain is attained when employing PC on top of AM in OFDM networks, it is important to note that NRSs forward the overheard transmissions using their original AM modes which are not selected based on the NRS-WT link. The main idea of the proposed algorithm is to allocate the requested data segment the proper subchannel and the proper amount of power to meet the target SINR of its AM mode, based on the medium access interference information. Preserving the AM modes of overheard transmissions simplifies the coordination between the NRS and the assisted WT. Moreover, conventional combining techniques such as selection or MRC can simply be invoked at the WT side. The two possible approaches to grouping the selected subcarriers are described as 'subchannel packing' and 'nopacking'. Figure 2 shows how the subchannel packing is done through example of using three subchannels on the FRS-WT link each possibly of a different AM mode while the erroneous subcarriers (shown in darker shades) are identified at the WT and the overhearing NRS. During the following BS sub-frame, the NRS forwards the subcarriers it reliably detected out of those requested by the WT. Given the shown medium access instant, the NRS acquired two reuse subchannels with the least

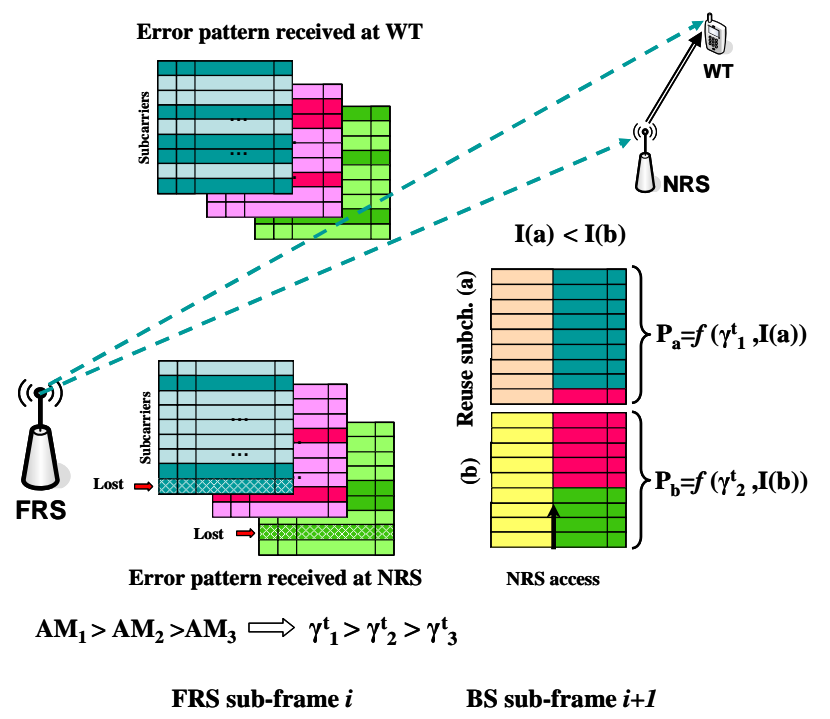

Fig. 2. NRS power-subchannel allocation for the case where the least possible number of subchannels is used, given the instant of the NRS medium access. interference observed. For the no-packing mechanism, more reuse subchannels are likely used (e.g., 3 instead of 2 as shown in Figure 3). The power calculation, however, is done similarly yet some subcarriers of low target SINR will enjoy a higher SINR if packed with a subcarrier group of high target SINR. While the packing approach has the advantage of utilizing least number of subchannels, its total power consumption has to be investigated along with its physical layer viability.

In both approaches, $\mathrm{NRS}_{k}$ transmit power on an acquired subchannel $\hat{n}$ is a function of the target SINR of the forwarded subcarrier group $i, \gamma_{k, i}^{t}$, the interference power $\mathbf{I}_{k}(\hat{n})$, as sensed by the NRS during its medium access, and the largescale pathloss $P_{L}$ which is the same across all the subchannels on that NRS-WT link and can be estimated and fedback over quite long time intervals. Saving the instantaneous feedback overhead from the WT reporting the subchannels of the NRSWT link, the NRS exploits such potentially high link quality and limited variability of the small-scale fading by assuming a certain fading margin $F_{M}$; This margin also accounts for the slight interference variability as seen by the WT. Let $P_{k, \hat{n}}$ denotes the transmit power of $\mathrm{NRS}_{k}$ (serving $\mathrm{WT}_{k}$ ) on subchannel $\hat{n}$ while $n_{0}$ is the noise power per subchannel. The joint power-subchannel allocation algorithm is the following:

1) The number of reuse subchannels to be acquired $N_{r}$ is determined based on the total number of subcarriers to be forwarded, the remaining portion of the BS subframe, and the packing approach employed.

2) All subchannels other than those assigned to the BS$\mathrm{WT}_{k}$ link, $\mathcal{N}-\mathcal{N}_{0 \rightarrow k}$, are sorted in ascending order of their observed interference power and the first $N_{r}$ subchannels are acquired, i.e., $\mathbf{I}_{k}(\hat{1})<\ldots<\mathbf{I}_{k}(\hat{n})<$ $\ldots<\mathbf{I}_{k}\left(\hat{N}_{r}\right)$.

3) The subcarrier groups to be forwarded are sorted in descending order of their target SINRs computed from the spectral efficiencies of the AM modes as $\gamma_{k, i}^{t}=$ $-\left(2^{\eta_{i}}-1\right) \ln \left(5 P_{e}\right) / 1.5$.

4) The first unscheduled group of subcarriers with the highest target $\gamma_{k, i}^{t}$ are scheduled on the first unassigned subchannel $\hat{n}$ which has the least interference $\mathbf{I}_{k}(\hat{n})$. 


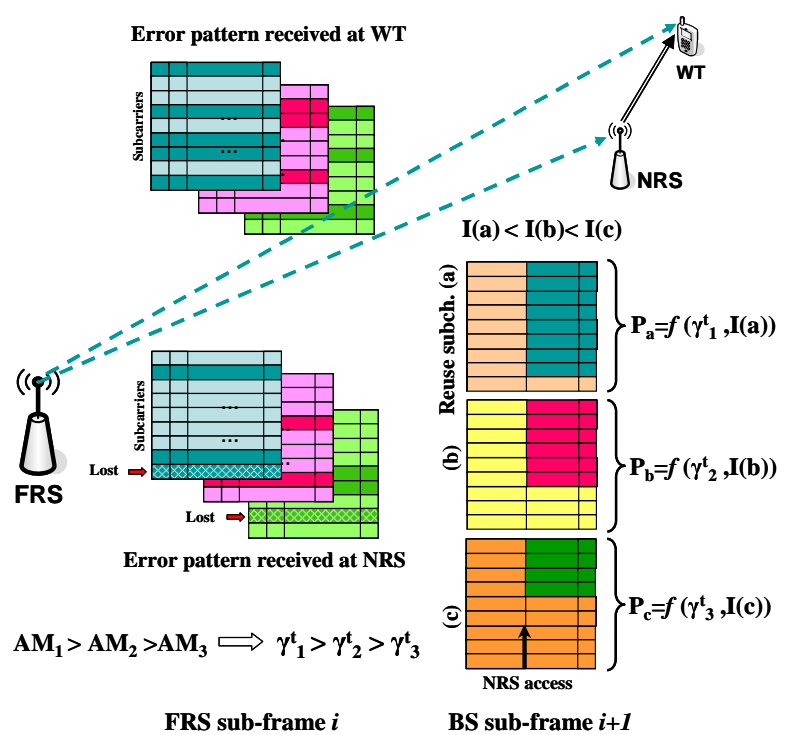

Fig. 3. NRS power-subchannel allocation for the case where no packing is employed and potentially more subchannels are used.

5) If the subchannel packing approach is employed and the subchannel is not fully loaded, more subcarriers from the next quality levels, i.e., $\gamma_{k, i+1}^{t}, \gamma_{k, i+2}^{t}, \ldots$, are packed into the subchannel (see Fig. 2). Otherwise, the subchannel is left partially loaded (see Fig. 3).

6) $P_{k, \hat{n}}$ is calculated in $\mathrm{dBm}$ using the following formula;

$P_{k, \hat{n}}=\min \left\{P_{\mathrm{k}}^{\mathrm{rem}}, 10 \log _{10}\left(\gamma_{k, i}^{t}\left(\mathbf{I}_{k}(\hat{n})+n_{0}\right)\right)+F_{M}+P_{L}\right\}$, where $P_{\mathrm{k}}^{\mathrm{rem}}$ is the remaining amount of the total power available at $\mathrm{NRS}_{k}, P_{\text {nom }}$. Therefore, $P_{\mathrm{k}}^{\text {rem }}$ is reduced thereafter by the calculated amount $P_{k, \hat{n}}$.

7) The steps 4) to 6) are repeated for each of the unassigned subchannels.

Allocating the most SINR-demanding subcarrier group the subchannel with the least interference results in the least amount of transmit power and hence the joint algorithm attempts to achieve the target SINRs and BER in an iterative manner with minimal total power expended and CCI caused. In the reference 'NoPC' NRS-augmented schemes proposed in [7], the subchannel transmit power $P_{\mathrm{k}}^{\max }$ is set to a fixed level equal to the total available power, $P_{\text {nom }}$ divided by $N$.

\section{Numerical Results}

The simulated cellular network consists of 19 hexagonal cells enhanced with 3 or 6 FRSs placed at a distance of 0.65 of the cell radius from the BS and with a uniform angular spacing. The distance between two adjacent BSs is $1 \mathrm{Km}$. The NRS is placed randomly within a distance of at most $10 \mathrm{~m}$ from the WT. NRS location, user location, and shadowing are regenerated each drop to collect statistics. Users are uniformly distributed within the cell area. Time-frequency correlated small-scale fading is assumed for all simulated links. Independent lognormal shadowing is considered for all links. According to most shadowing spatial correlation models as applied to such network geometry, the same shadowing realization is applied to an FRS-WT link and its associated FRS-NRS link, for both serving and interfering FRSs. A list of channel and system parameters are given in Table I. The path-loss model for the links as a function of the distance
TABLE I

SYSTEM AND SIMULATION PARAMETERS.

\begin{tabular}{|c|c|}
\hline \hline Parameter & Value \\
\hline BS-BS distance & $1 \mathrm{Km}$ \\
\hline User min. close-in distance to BS & $35 \mathrm{~m}$ \\
\hline BS Tx. antenna gain & $15 \mathrm{~dB}$ \\
\hline FRS Tx. antenna gain & $10 \mathrm{~dB}$ \\
\hline WT Rx. antenna gain & $0 \mathrm{~dB}$ \\
\hline Shadowing st. dev. on user and interfering links (NLOS) & $8.9 \mathrm{~dB}$ \\
\hline Shadowing st. dev. on NRS-WT links (LOS) & $1.5 \mathrm{~dB}$ \\
\hline Carrier frequency & $2.5 \mathrm{GHz}$ \\
\hline Total bandwidth & $20 \mathrm{MHz}$ \\
\hline User mobility & $10 \mathrm{Km} / \mathrm{hr}$ \\
\hline Number of taps in the PDP & 6 \\
\hline Channel sampling time = TDD frame length & $5 \mathrm{msec}$ \\
\hline Downlink : Uplink ratio & $2: 1$ \\
\hline DL Tx. time in OFDM data symbols & $24 \mathrm{symbols}$ \\
\hline OFDM subcarrier bandwidth & $10.9375 \mathrm{KHz}$ \\
\hline OFDM symbol duration & $102.86 \mathrm{sec}$ \\
\hline Subchannel width & $18 \mathrm{subcarriers}$ \\
\hline Noise power density at Rx. nodes & $-174 \mathrm{dBm} / \mathrm{Hz}$ \\
\hline Power control fading margin & $3 \mathrm{~dB}$ \\
\hline BS total Tx. power & $46 \mathrm{dBm}$ \\
\hline RS total Tx. power & $37 \mathrm{dBm}$ \\
\hline NRS total Tx. power & $23 \mathrm{dBm}$ \\
\hline \hline
\end{tabular}

(in meters) is given as $P_{L}=38.4+A \log _{10}(d) \mathrm{dB}$. For the serving NRS-WT links, $A=20.0$, and $A=35$ otherwise. The minimum rate requirement is set to $250 \mathrm{Kbps}$ and the target BER is $10^{-3}$ for all users. The distributed RRA scheme and only the no-packing approach are considered. We define the power saving gain as the ratio of the total NRS transmit power without PC (NoPC) to that with PC.

$$
P S_{\text {tot }}=10 \log _{10}\left(\frac{P_{\text {to }}^{\mathrm{NoPC}}}{P_{\text {tot }}^{\mathrm{PC}}}\right)[\mathrm{dB}] .
$$

Figure 4 shows the CDF plots of the power saving gain achieved by the PC scheme with 15 WTs/cell using 3 and 6 FRSs. The wide region to the right of the vertical line at $0 \mathrm{~dB}$ represents the saving region where the PC scheme allocates less total power for the NRS transmission than the NoPC scheme which uses the same number of reuse subchannels and a power of $2.91 \mathrm{dBm} / \mathrm{subchannel}$. It can be observed that when 3 FRSs are deployed per cell, the scheme with PC achieves substantial power savings as compared to the NoPC scheme for $80 \%$ of the time, while it requires higher total power for $20 \%$ of the time based on the target SINRs, interference, and pathloss conditions. The mean saving gain in that case is around $28 \mathrm{~dB}$. With 6 FRSs, less saving gains, but still substantial, are achieved for $71 \%$ of the time with a mean value of around $21 \mathrm{~dB}$. Having maintained the same large-scale and small-scale fading statistics for the $M=3$ and $M=6$, the WT-based routing and the geographical deployment of the FRSs along with the uniform user distribution result, more often, in larger distances between the WTs and their serving FRSs in the case of 3 FRSs/cell. This implies that FRSs will more often adopt lower AM modes, which require less target SINRs than the case of 6 FRSs/cell. Since the NRS forwards the overheard FRS transmission using the same AM mode, less transmit power levels are allocated with 3 FRSs than with 6 FRSs. In a way this is a power-throughput trade-off since deploying more FRSs improves user throughput [7] yet less NRS power savings are anticipated. It is important to note that our PC mechanism is designed to satisfy the requirement of the 


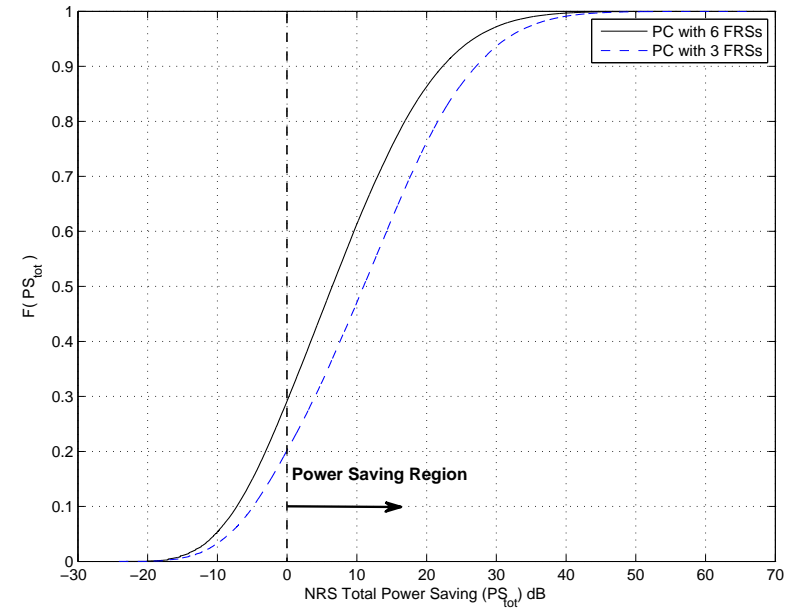

Fig. 4. CDF plots of the total power saving (gain) achieved by the RRM scheme with PC for $15 \mathrm{WTs} / \mathrm{cell}$.

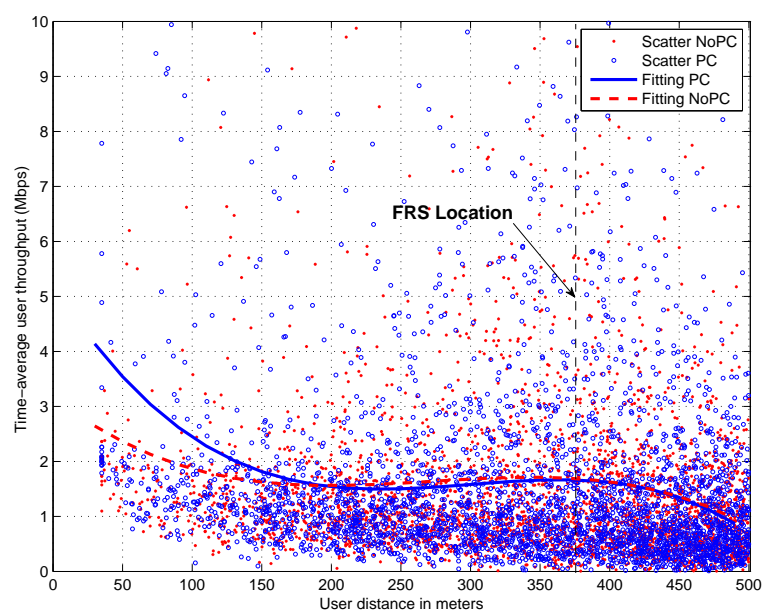

Fig. 5. Scatter of user time-average throughput for the schemes with 15 WTs and 3 FRSs. Curve fittings represent the distance conditional means.

transmissions with the highest AM modes first. Meaning that, when loss is inevitable due to the lack of power, only the last transmissions in the routine, with the least number of loaded bits, would be risked. This worst-case defense strategy results in the least throughput loss whenever it is inevitable. The CCI due to the NRS channel reuse may only affect the BS-dWT, BS-FRS, or other NRS-WT links. However, FRSs are deployed at strategic locations with good LOS communications and highly directive antennas to the BS; their feeder links are much immune to CCI than the dWTs. Figure 5 shows the scatter plot of user time-averaged throughput versus user distance from the BS for a network with 3 FRSs. As expected, the PC scheme outperforms the reference NoPC scheme within only the vicinity of the BS where dWTs are most likely located and subjected to the CCI due to NRS intra-cell reuse. Observing the vicinity of FRSs suggests that the mutual CCI between NRSs is negligible in the original scheme (NoPC) due to the opportunistic medium access technique and therefore, no further gain is achieved by reducing the NRS transmit power most of the time. Figure 6 shows the CDF plots of the instantaneous throughput of dWTs for the PC and NoPC schemes. It can be seen that a significant throughput gain is achieved through improving the CCI situation of the dWTs.

\section{CONCLUSIONS}

We devised a novel joint power and subchannel allocation algorithm employing an open-loop APC mechanism for the

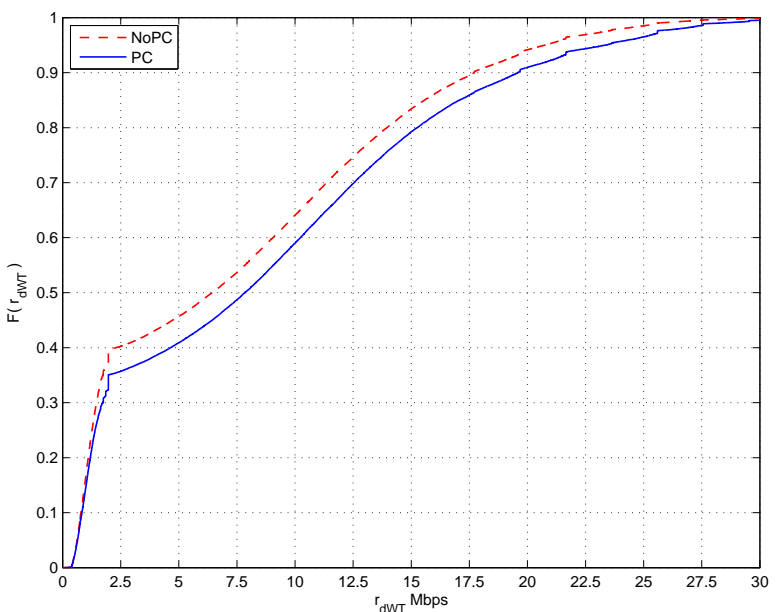

Fig. 6. CDF plots of the instantaneous throughput of dWTs for the PC and NoPC schemes with 15 WTs and 6 FRSs/cell.

new OFDMA-based nomadic-augmented fixed relay networks. The results before hand established a proof of concept for the proposed PC mechanisms. Substantial power savings are achieved at the NRS resulting in extended battery life and less frequent recharging. This smoothly integrated add-on feature to our autonomous NRS medium access technique brings around the timely environmental concerns in designing future wireless communication networks. The value of such an efficient add-on is further emphasized given that the APC mechanism requires no feedback from network nodes and relies on the available medium access information. It has been observed in our illustrative system that PC at the NRSs can further improve the throughput of the BS's directly connected users whereas no throughout gains are achieved for the relayed users due the inherent CCI spreading in the NRS opportunistic medium access. A power-throughput trade-off is addressed in terms of the number of deployed FRSs. We thus further establish our pioneering techniques for realizing the concept of NRS-augmented networks.

\section{REFERENCES}

[1] D. Soldani and S. Dixit, "Wireless relays for broadband access," IEEE Communications Magazine, 46(3), pp. 58-66, March 2008.

[2] IEEE 802.16, "Mobile multihop relay study group recommendations for the scope and purpose of the mobile multihop relay task group," 11 November 2005, http:www.ieee802.org/16/sg/mmr/contrib/C80216mmr05_032.pdf.

[3] J. Zander, "Distributed cochannel interference control in cellular radio systems," IEEE Transactions on Vehicular Technology, 41(3), pp. 305311, August 1992.

[4] S. Ulukus and R. Yates, "Stochastic power control for cellular radio systems," IEEE Transactions on Communications, 46(6), pp. 784-798, June 1998.

[5] M. Pischella and J.-C. Belfiore, "Power control in distributed cooperative OFDMA cellular networks," IEEE Transactions on Wireless Communications, 7(5), pp. 1900-1906, May 2008.

[6] Online article, "BARCELONA MWC 2009: The restructuring of a sector," Mobile World Congress, Barcelona, March 2009. Available at: http://sociedaddelainformacion.telefonica.es/jsp/articulos/

[7] M. Salem, A. Adinoyi, H. Yanikomeroglu, and Y.-D. Kim, "Radio resource management in OFDMA-based networks enhanced with fixed and nomadic relays," to appear in IEEE Wireless Communications \& Networking Conference, April 2010.

[8] X. Qiu and K. Chawla, "On the performance of adaptive modulation in cellular systems," IEEE Transactions on Communications, 47(6), pp. 884-895, June 1999.

[9] B. Babadi and V. Tarokh, "A distributed asynchronous algorithm for spectrum sharing in wireless ad hoc networks," Annual Conference on Information Sciences and Systems, pp. 831-835, March 2008. 